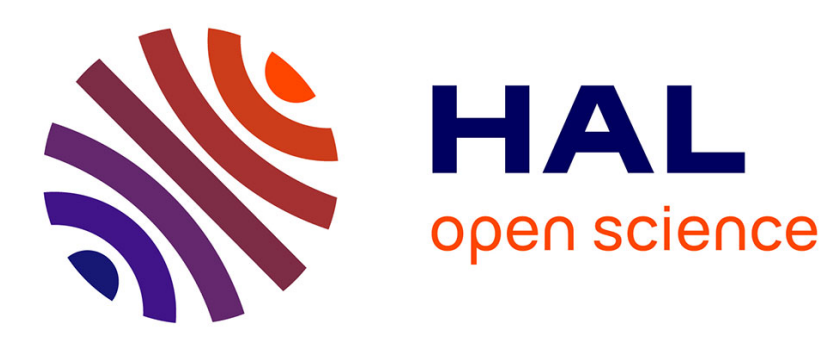

\title{
Integrated Digital Image Correlation for the Evaluation and Correction of Optical Distortions
}

John-Eric Dufour, François Hild, Stéphane Roux

\section{To cite this version:}

John-Eric Dufour, François Hild, Stéphane Roux. Integrated Digital Image Correlation for the Evaluation and Correction of Optical Distortions. Optics and Lasers in Engineering, 2014, 56, pp.121-133. 10.1016/j.optlaseng.2013.12.015 . hal-00947401

\section{HAL Id: hal-00947401 \\ https://hal.science/hal-00947401}

Submitted on 15 Feb 2014

HAL is a multi-disciplinary open access archive for the deposit and dissemination of scientific research documents, whether they are published or not. The documents may come from teaching and research institutions in France or abroad, or from public or private research centers.
L'archive ouverte pluridisciplinaire HAL, est destinée au dépôt et à la diffusion de documents scientifiques de niveau recherche, publiés ou non, émanant des établissements d'enseignement et de recherche français ou étrangers, des laboratoires publics ou privés. 


\title{
Integrated Digital Image Correlation for the Evaluation and Correction of Optical Distortions
}

\author{
John-Eric Dufour ${ }^{\mathrm{a}}$, François Hild ${ }^{\mathrm{a}, *}$, Stéphane Roux $^{\mathrm{a}}$ \\ ${ }^{a}$ Laboratoire de Mécanique et Technologie (LMT-Cachan) \\ ENS Cachan / CNRS / UPMC / PRES UniverSud Paris \\ 61 Avenue du Président Wilson, F-94235 Cachan Cedex, France
}

\begin{abstract}
Optical aberrations are one of the biases affecting images. Their correction can be performed as a transformation of the image. In this paper, it is proposed to use digital image correlation to calculate the distortion fields. A correlation calculation is performed between a known numerical pattern and a picture of the same pattern printed or etched on a plate. This new procedure is applied to determine the distortion fields of a camera lens. First, a parametric description of the distortion field is used to reduce the degrees of freedom required to describe optical distortions. Second, a non-parametric model based upon splines is used. The distortion fields found by both methods are compared. A resolution analysis is performed for two different procedures (removing the lens between pictures or not). The standard displacement resolution is found to be of the order of $10^{-2}$ pixel in the first case, and $2.5 \times 10^{-3}$ pixel in the second case. Keywords: DIC; Integrated approach; Optical distortions; Resolution.
\end{abstract}

\footnotetext{
${ }^{*}$ Corresponding author. Phone: +331474021 92, Fax: +33147402240.

Email addresses: john-eric.dufour@lmt.ens-cachan.fr (John-Eric Dufour), francois.hild@lmt.ens-cachan.fr (François Hild), stephane.roux@lmt.ens-cachan.fr (Stéphane Roux)
} 


\section{Introduction}

Digital image correlation consists of the registration of pictures taken by cameras. The mechanism of taking a picture with a digital camera induces some differences between the ideal geometrical projection of the real scene and the acquired picture. As the definition of the camera sensors increases, the main source of errors is now due to lenses generating geometrical aberrations resulting in a distortion field representing the displacement field between the real scene and the shot one. Digital image correlation uses pictures to measure displacements, therefore these systematic errors bias the measurements $[1,2,3$, $4,5,6,7]$. That is the reason why a correction of these distortions has to be performed so that measurements become quantitative and bias-free.

Several procedures have already been proposed to measure and correct distortions so as to restore the geometrical fidelity of images. Many of these methods use geometrical calibration targets (e.g., grids, checkered surfaces) to perform the correction. The latter consists of linear coordinate transformations for non-parametric ${ }^{1}$ descriptions with no relationship to the physics of distortion phenomena. Other techniques use similar methods to obtain stereocorrelation calibration matrices and distortion correction procedures $[8,9,11,4,5]$. This allows a single calibration step to achieve both objectives. Another strategy consists of using a parametric description of the distortion field. The latter is extracted and then fitted to obtain a set of parameters $[2,1,12]$. This method generally uses grids to obtain the distortion field but implies a modification of

\footnotetext{
${ }^{1}$ The terminology "parametric" refers to a model-based representation of the measurement so that the parameters can be directly interpreted as the characteristics of a physical model. In contrast a discretization based on a mathematically convenient basis without direct physical interpretation will be called "non-parametric" to comply with a common convention $[8,9,10$, $11,5]$.
} 
the image, which is a non-linear transformation (addition of a new field to the image). Some approaches use both nonparametric model and nonlinear transformation. For instance, a spline-based description [12] can be used to correct for distortions. With these methods, only a few reference points are extracted from the whole pictures of the calibration target. The most used registration techniques are based on geometrical elements (for example circles or intersecting lines) to determine remarkable points on a target using e.g. Harris' corner detector [13]. Correlation-based approaches are another alternative $[12,5]$ used herein. In contrast with the previous method, a dense measurement of an apparent displacement field is performed herein with a uniform measurement over a region of interest, and it does not rely on interpolation or regression procedures in between remarkable points.

It is proposed to use an integrated approach to DIC directly considering a parametric description of the distortion field. Although most methods are based upon regular geometrical targets, the present analysis is based on a printed or etched random pattern to extract the distortions from the whole picture. The integrated approach allows us to directly extract the parameters from the DIC analysis without any additional interpolation. It can be used in addition to a spline basis to calculate the parameters of a spline-based correction of distortion.

The outline of the paper is as follows. First, the concept of optical distortion is introduced by defining the underlying physical biases. Then the method to evaluate distortions is presented. It is based on an integrated DIC approach. A resolution analysis is also performed by both analytical and experimental approaches. Last, a spline-based method and its resolution analysis are compared for two different basis orders. 


\section{Optical Distortions}

In the sequel, the main optical distortions and the mathematical model to account for these biases are introduced.

\subsection{Physics of Distortions}

Optical distortions are mainly caused by imperfections of cameras and their optics. Different kinds of defaults generate optical distortions. Thickness of the lenses and alignments in the optical system are generally approximations of a camera model and are not perfect. Several types of optical biases are generally identified $[2,8,5]$ and only three of them are listed and studied:

- Radial distortions are due to the paraxial approximation. When light comes into the system with a large angle from the optical axis, the Gaussian approximation of its path does not represent the reality. These rays do not hit the CCD (or CMOS) sensor at the location predicted by the model, thereby causing a distortion [1].

- Decentering distortions are caused by the lack of coaxiality of the center of the lenses composing the optics. In an aligned optical system, rays traveling along the center of the lenses are not deviated. If the different centers are not coaxial, all rays deviate. Although an 'apparent' optical axis can be defined to take into account the whole system, it does not coincide with the optical center predicted by the perfect camera model [1].

- Thin prism distortions are due to the fact that the parallelism of the lens between each other and with the CCD or CMOS sensor is not completely satisfied. This last distortion is also known as tilt distortion [1]

Optical biases can be described as an apparent displacement field between an image taken with a real camera and a perfect image of the same scene. 


\subsection{Apparent Motions}

For each distortion described above, a mathematical model exists. It accounts for the fact that the true pixel location $\left(x_{r}, y_{r}\right)$ is biased and therefore has 'moved' by an apparent displacement $\left(\delta_{x}, \delta_{y}\right)$ so that its position in the picture is $\left(x_{r}+\delta_{x}, y_{r}+\delta_{y}\right)$. Correcting optical distortions consists of determining the apparent motions $\left(\delta_{x}, \delta_{y}\right)[1,2]$. To correct for distortions, the distorted image has to be corrected to bring it back to the reference (i.e., undistorted) configuration. This is precisely what is performed in the registration operation in any DIC code (see below). The only difference is that the displacement field $\left(\delta_{x}\left(x_{r}, y_{r}\right), \delta_{y}\left(x_{r}, y_{r}\right)\right)$ is known since the distortion components have been determined, as shown in the sequel. Consequently, correcting for distortions consists of calculating $\tilde{g}\left(x_{r}, y_{r}\right)=g\left(x_{r}+\delta_{x}\left(x_{r}, y_{r}\right), y_{r}+\delta_{y}\left(x_{r}, y_{r}\right)\right)$ for any considered pixel location $\left(x_{r}, y_{r}\right)$. Since $g$ is known at the pixel locations $\left(x_{r}, y_{r}\right)$, $g\left(x_{r}+\delta_{x}\left(x_{r}, y_{r}\right), y_{r}+\delta_{y}\left(x_{r}, y_{r}\right)\right)$ will require evaluations at non integer positions. Thus, gray level interpolations are needed, which are identical to those used in the DIC procedure.

Radial Distortions. The radial distortions are related to the series of odd powers of the radial coordinate of a pixel. They are described as an infinite series $[1,2]$

$$
\begin{aligned}
& \delta_{x}^{R}\left(x_{r}, y_{r}\right)=x \sum_{k=1}^{\infty} r_{k}\left(x_{r}^{2}+y_{r}^{2}\right)^{k} \\
& \delta_{y}^{R}\left(x_{r}, y_{r}\right)=y \sum_{k=1}^{\infty} r_{k}\left(x_{r}^{2}+y_{r}^{2}\right)^{k}
\end{aligned}
$$

where $\delta_{x}^{R}, \delta_{y}^{R}$ denote the apparent displacements due to radial distortions, and $r_{k}$ are the corresponding (unknown) amplitudes.

Decentering Distortions. This model represents the influence of having an apparent optical center, which is different from the assumed one. It is also ex- 
pressed as an infinite series $[1,2]$

$$
\begin{aligned}
& \delta_{x}^{D}\left(x_{r}, y_{r}\right)=\left(2 x_{r} y_{r} \cos \phi_{0}-\left(3 x_{r}^{2}+y_{r}^{2}\right) \sin \phi_{0}\right) \sum_{k=1}^{\infty} s_{k}\left(x_{r}^{2}+y_{r}^{2}\right)^{k-1} \\
& \delta_{y}^{D}\left(x_{r}, y_{r}\right)=\left(2 x_{r} y_{r} \sin \phi_{0}-\left(x_{r}^{2}+3 y_{r}^{2}\right) \cos \phi_{0}\right) \sum_{k=1}^{\infty} s_{k}\left(x_{r}^{2}+y_{r}^{2}\right)^{k-1}
\end{aligned}
$$

where $\delta_{x}^{D}, \delta_{y}^{D}$ denote the apparent displacements due to decentering distortions, $s_{k}$ the corresponding (unknown) amplitudes, and $\phi_{0}$ the angular position of the apparent center in the picture.

Thin Prism Distortions. This bias is related to the series of even powers of the radial coordinate. An infinite series $[2,1]$ is also utilized

$$
\begin{aligned}
& \delta_{x}^{P}\left(x_{r}, y_{r}\right)=-\sin \phi_{0} \sum_{k=1}^{\infty} t_{k}\left(x_{r}^{2}+y_{r}^{2}\right)^{k} \\
& \delta_{y}^{P}\left(x_{r}, y_{r}\right)=\cos \phi_{0} \sum_{k=1}^{\infty} t_{k}\left(x_{r}^{2}+y_{r}^{2}\right)^{k}
\end{aligned}
$$

where $\delta_{x}^{P}, \delta_{y}^{P}$ are the apparent displacements due to prismatic distortions, $t_{k}$ the corresponding (unknown) amplitudes.

In this study, we will use a first order expansion $(k=1)$ of the previous expressions

$$
\begin{aligned}
& \delta_{x}^{R}\left(x_{r}, y_{r}\right)=r_{1} x_{r}\left(x_{r}^{2}+y_{r}^{2}\right) \\
& \delta_{y}^{R}\left(x_{r}, y_{r}\right)=r_{1} y_{r}\left(x_{r}^{2}+y_{r}^{2}\right) \\
& \delta_{x}^{S}\left(x_{r}, y_{r}\right)=2 d_{2} x_{r} y_{r}-d_{1}\left(3 x_{r}^{2}+y_{r}^{2}\right) \\
& \delta_{y}^{S}\left(x_{r}, y_{r}\right)=2 d_{1} x_{r} y_{r}-d_{2}\left(3 x_{r}^{2}+y_{r}^{2}\right) \\
& \delta_{x}^{P}\left(x_{r}, y_{r}\right)=p_{1}\left(x_{r}^{2}+y_{r}^{2}\right) \\
& \delta_{y}^{P}\left(x_{r}, y_{r}\right)=p_{2}\left(x_{r}^{2}+y_{r}^{2}\right)
\end{aligned}
$$

This restriction to the first orders allows us to keep a few unknowns for the system (i.e., 5 coefficients) while modeling the most salient features. This is the standard hypothesis made to model distortions $[1,2,8,5]$ 


\section{Distortion Evaluation via Integrated-DIC}

In this section, Digital Image Correlation $[3,5,14]$ will be used. Contrary to the common practice of DIC, which is local (i.e., subset-based), the whole ROI will be analyzed as a single interrogation window. Two different approaches will be considered. First, when a non-parametric distortion model is assumed, a spline-based displacement basis is chosen. This type of approach was already used to analyze beam bending [15] and wood sample compression [16]. An alternative is to resort to so-called integrated-DIC $[17,18]$ in which the sought displacement field is directly given by, say, Equation (4). There is therefore no need to re-project the measured displacement field.

To perform DIC analyses, several images are required. Let us first consider an image generated numerically as a random pattern of black dots on a white field. This picture is considered as the reference configuration $f(\boldsymbol{x})$. It corresponds to the model of the calibration target used hereafter. This reference can be printed (or etched) as a "physical" calibration target and pictured by a camera. This second image has experienced optical biases due to the imperfections of the objective lenses of the camera. This corresponds to image $g(\boldsymbol{x})$ in the "deformed" — or rather "distorted" — configuration. It is to be stressed that the measured distortions consist of the optical distortion of the imaging device as well as the printing or etching distortion. The partition of the global distortions between these two sources will not be discussed herein (see Ref. [19] on that issue). However, the technology of printing or etching may not share the same type of distortions as the optical ones, in which case some distortion modes may be considered as resulting primarily from one source or another. For instance, if the printing is carried out with a high fidelity ink-jet printer, one of the main sources of distortion is a line to line repositioning, which is not expected for a camera lens. This is in line with the philosophy of using a 
parametric approach. However, if it cannot be proven that the optical modes are absent in the printing process, this separation cannot be performed in a definitive way.

In this paper, two different calibration targets have been used. The first one has been printed on an aluminum sheet (Figure 1(b)) from a reference picture shown in Figure 1(a). The second one has been etched on a steel plate (Figure 2(b)) from another reference picture (Figure 2(a)). The two references are composed of black dots randomly distributed on a white background, their location is not exactly the same in both cases. The plane is partitioned into equally sized square elements and one disk is randomly positioned within each square. The center and the diameter of these dots are therefore known.

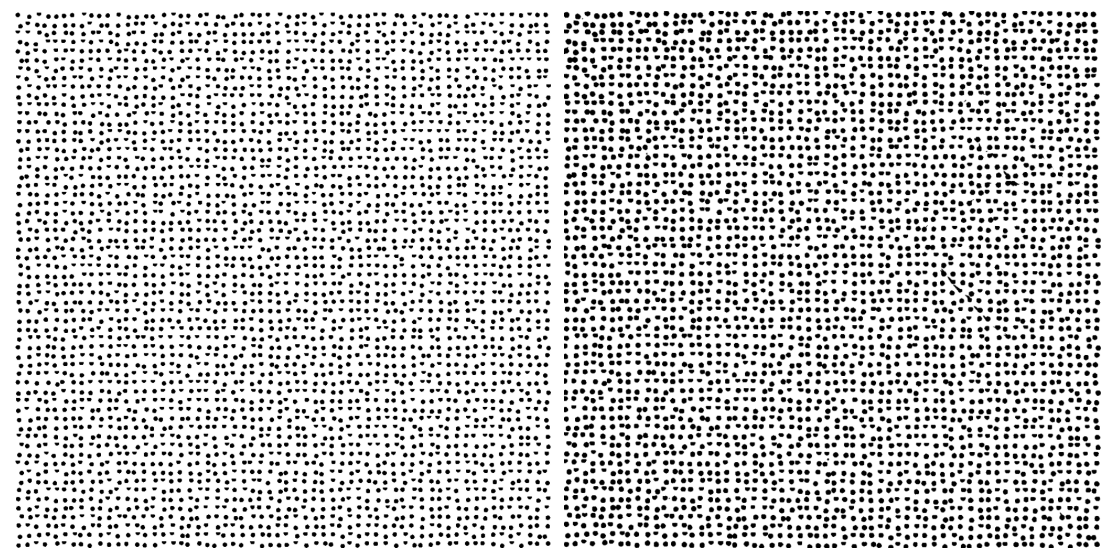

(a)

(b)

Figure 1: Numerical reference (a) and picture of the printed calibration target (b). It can be noted that there are some defaults due to the printer

Contrary to standard calibration targets $[8,10,4,5]$, the calibration procedure is based on a random pattern. It allows us to use digital image correlation instead of a geometrical approach to correct for distortions. This numerical reference is then printed onto an aluminum sheet or laser etched on a stainless steel plate that was initially burnished. To determine the orientation of the 


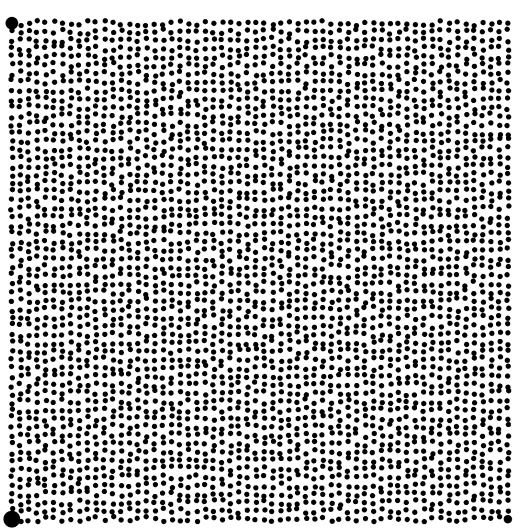

(a)

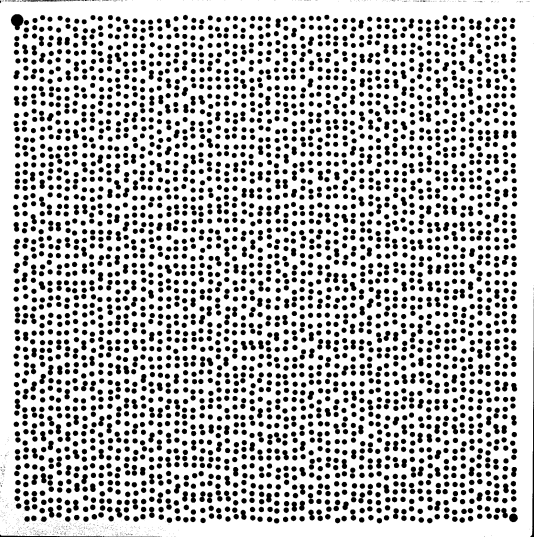

(b)

Figure 2: Numerical reference (a) and picture of the etched calibration target (b). It can be noted that the large dot on the lower left corner was not etched

target 3 larger dots are marked in the corners (Figure 2). The etched target used herein is a $150 \times 150 \mathrm{~mm}^{2}$ plate with $1.46-\mathrm{mm}$ diameter dots. The printed target is a $150 \times 150 \mathrm{~mm}^{2}$ plate with 1 -mm diameter dots.

\subsection{DIC Algorithm}

DIC consists of satisfying as best as possible the gray level conservation

$$
f(\boldsymbol{x})=g(\boldsymbol{x}+\boldsymbol{u}(\boldsymbol{x}))
$$

by minimizing the sum of squared differences

$$
\eta^{2}=\sum_{\mathrm{ROI}}[\boldsymbol{g}(\boldsymbol{x}+\boldsymbol{u}(\boldsymbol{x}))-f(\boldsymbol{x})]^{2}
$$

over the considered Region of Interest (ROI) with respect to the unknown displacement field $\boldsymbol{u}$. To estimate $\boldsymbol{u}$, a modified Newton scheme is used in which the linearized functional $\eta_{\operatorname{lin}}^{2}$ is considered

$$
\eta_{\text {lin }}^{2}=\sum_{\mathrm{ROI}}[g(\boldsymbol{x}+\tilde{\boldsymbol{u}}(\boldsymbol{x}))+\delta \boldsymbol{u}(\boldsymbol{x}) \cdot \boldsymbol{\nabla} f(\boldsymbol{x})-f(\boldsymbol{x})]^{2}
$$


where $\tilde{\boldsymbol{u}}$ is the current estimate of the sought displacement field, and $\delta \boldsymbol{u}$ is the estimated correction to be applied.

At this point, the displacement field $\boldsymbol{u}(\boldsymbol{x})$ can be decomposed over a set of trial fields $\psi_{n}(\boldsymbol{x})$

$$
\boldsymbol{u}(\boldsymbol{x})=\sum_{n} a_{n} \boldsymbol{\psi}_{n}
$$

where $a_{n}$ are the unknown amplitudes. As mentioned above, two different bases will be tested in the following studies. First, when a parametric description of the distortions is used, Equation (4) defines displacement fields that can be considered directly in the present setting. Second, a non-parametric description is also possible (e.g., spline-based) of the sought displacement field.

The linearized functional $\eta_{\text {lin }}^{2}$ is thus rewritten as a function of the unknown amplitudes so that its minimization leads to linear systems in terms of the corrections $\delta a_{m}$

$$
M_{m n} \delta a_{n}=b_{m}
$$

with

$$
\begin{aligned}
M_{m n} & =\sum_{\text {ROI }}\left(\boldsymbol{\psi}_{m} \cdot \boldsymbol{\nabla} f\right)(\boldsymbol{x})\left(\boldsymbol{\psi}_{n} \cdot \boldsymbol{\nabla} f\right)(\boldsymbol{x}) \\
b_{m} & =\sum_{\mathrm{ROI}}\left[f(\boldsymbol{x})-g\left(\boldsymbol{x}+\sum_{n} \tilde{a}_{n} \boldsymbol{\psi}_{n}(\boldsymbol{x})\right)\right]\left(\boldsymbol{\psi}_{m} \cdot \boldsymbol{\nabla} f\right)(\boldsymbol{x})
\end{aligned}
$$

where $\{\tilde{\boldsymbol{a}}\}$ are the current estimates of the displacement amplitudes. The minimization stops when reaching a gray level residual less than to $1 \%$ of the dynamic range of the reference picture or when the RMS difference between two consecutive solutions $\{\tilde{\boldsymbol{a}}\}$ is less than $10^{-3}$ pixel. This value can be justified with the resolution analysis performed in Section 4.1. A linear interpolation of the gray levels is used in the proposed examples and the picture gradient is calculated using centered differences for inner pixels and forward and backward differences on the edges of the ROI. 


\subsection{Resolution Analysis}

The resolution is defined as the 'smallest change, in the value of a quantity being measured by a measuring system, that causes a perceptible change in the corresponding indication. The resolution of a measuring system may depend on, for example, noise (internal or external) [...]' [20]. A reference picture is considered and the sensitivity of the measured degrees of freedom $\delta a_{n}$ to noise associated with image acquisition is assessed. In the present case, only the deformed image is affected by acquisition noise.

The noise is modeled by a random variable $\zeta$, which is added to $g$. It is assumed that $\zeta$ is a Gaussian white noise, of zero mean, and covariance $\sigma^{2} \boldsymbol{I}(\boldsymbol{x}, \boldsymbol{y})$, where $\boldsymbol{x}$ and $\boldsymbol{y}$ denote the locations of the pixels, $\sigma^{2}$ the variance, and $\boldsymbol{I}$ the Kronecker matrix. With these hypotheses, matrix $[\boldsymbol{M}]$ is unaffected by noise, but the right hand side term $\{\boldsymbol{b}\}$ is modified by an increment [18]

$$
\delta b_{m}=\sum_{\boldsymbol{v}}\left(\boldsymbol{\psi}_{m} \cdot \nabla f\right)(\boldsymbol{v}) \zeta(\boldsymbol{v})
$$

whose average is thus equal to 0 , and its covariance reads

$$
\begin{aligned}
C\left(\delta b_{m}, \delta b_{n}\right) & =\sigma^{2} \sum_{\boldsymbol{v}} \sum_{\boldsymbol{w}}\left(\boldsymbol{\psi}_{m} \cdot \nabla f\right)(\boldsymbol{v}) \delta(\boldsymbol{v}, \boldsymbol{w})\left(\boldsymbol{\psi}_{n} \cdot \boldsymbol{\nabla} f\right)(\boldsymbol{w}) \\
& =\sigma^{2} M_{m n}
\end{aligned}
$$

where $M_{m n}$ are the components of matrix [ $\left.\boldsymbol{M}\right]$ to be inverted in the DIC problem [see Equation (10)], and $\delta(.,$.$) is Kronecker's delta function. By linearity [see$ Equation (10)], the mean value of $\delta a_{p}$ vanishes, and its covariance becomes

$$
C\left(\delta a_{p}, \delta a_{q}\right)=\sigma^{2} M_{p m}^{-1} M_{m n} M_{n q}^{-1}=\sigma^{2} M_{p q}^{-1}
$$

\subsection{Integrated DIC: DIC with Distortion Model}

Let us consider a basis of displacement fields directly deduced from the considered distortion model [Equation (4)]. Lower degree fields need to be added 
Table 1: Link between displacement fields and distortion parameters for dimensionless coordinates $(X, Y)$ and $\left(X_{r}, Y_{r}\right)$

\begin{tabular}{|c|c|c|}
\hline Parameter & Component along $\boldsymbol{e}_{x}$ & Component along $\boldsymbol{e}_{y}$ \\
\hline$u_{0}$ & 1 & 0 \\
$v_{0}$ & 0 & 1 \\
$u_{1 x}$ & $X$ & 0 \\
$v_{1 x}$ & 0 & $X$ \\
$u_{1 y}$ & $Y$ & 0 \\
$v_{1 y}$ & 0 & $Y$ \\
$d_{1}$ & $-\left(3 X_{r}^{2}+Y_{r}^{2}\right)$ & $2 X_{r} Y_{r}$ \\
$d_{2}$ & $2 X_{r} Y_{r}$ & $-\left(X_{r}^{2}+3 Y_{r}^{2}\right)$ \\
$p_{1}$ & $X_{r}^{2}+Y_{r}^{2}$ & 0 \\
$p_{2}$ & 0 & $X_{r}^{2}+Y_{r}^{2}$ \\
$r_{1}$ & $X_{r}\left(X_{r}^{2}+Y_{r}^{2}\right)$ & $\left.X_{r}^{2}+Y_{r}^{2}\right)$ \\
\hline
\end{tabular}

to account for rigid body motions between the two pictures, and magnification changes (see Appendix). Thus a basis containing eleven fields is obtained, corresponding to eleven unknown amplitudes. Let us note however that the distortion fields are naturally expressed in coordinates $\left(x_{r}, y_{r}\right)$ whose origin is the optical axis, whereas the additional fields are expressed in coordinates $(x, y)$ whose origin is the image center (as the optical axis is not known a priori). In the following it is more convenient to introduce a characteristic length $L$ (height or width expressed in number of pixels), and reduced coordinates $X \equiv x / L$ and $Y \equiv y / L$ (and equivalently with subscripted coordinates). Table 1 gives the expression of the different distortion fields and additional fields, where it is important to distinguish coordinates centered on the image or on the optical axis. With the present convention, the amplitudes of the different fields are all expressed in pixels. By considering these trial fields, the number of degrees of 
Table 2: Link between distortion parameters and un-centered distortion fields for dimension-

\begin{tabular}{|c|c|c|}
\hline less coordinates & $e_{x}$ & $e_{y}$ \\
\hline$u_{0}+\left(d_{1}+p_{1}-r_{1} X_{c}\right)\left(X_{c}^{2}+Y_{c}^{2}\right)+2 d_{1} X_{c}^{2}+2 d_{2} X_{c} Y_{c}$ & 1 & 0 \\
$v_{0}+\left(d_{2}+p_{2}-r_{1} Y_{c}\right)\left(X_{c}^{2}+Y_{c}^{2}\right)+2 d_{1} X_{c} Y_{c}+2 d_{2} Y_{c}^{2}$ & 0 & 1 \\
$u_{1 x}+r_{1}\left(3 X_{c}^{2}+Y_{c}^{2}\right)-2 p_{1} X_{c}-6 d_{1} X_{c}-2 d_{2} Y_{c}$ & $X$ & 0 \\
$v_{1 x}+r_{1}\left(2 X_{c} Y_{c}\right)-2 p_{2} X_{c}-2 d_{1} Y_{c}-2 d_{2} X_{c}$ & 0 & $X$ \\
$u_{1 y}+r_{1}\left(2 X_{c} Y_{c}\right)-2 p_{1} Y_{c}-2 d_{1} Y_{c}-2 d_{2} X_{c}$ & $Y$ & 0 \\
$v_{1 y}+r_{1}\left(X_{c}^{2}+3 Y_{c}^{2}\right)-2 p_{2} Y_{c}-2 d_{1} X_{c}-6 d_{2} Y_{c}$ & 0 & $Y$ \\
$d_{1}-r_{1} X_{c}$ & $-\left(3 X^{2}+Y^{2}\right)$ & $2 X Y$ \\
$d_{2}-r_{1} Y_{c}$ & $2 X Y$ & $-\left(X^{2}+3 Y^{2}\right)$ \\
$p_{1}$ & $X^{2}+Y^{2}$ & 0 \\
$p_{2}$ & 0 & $X^{2}+Y^{2}$ \\
$r_{1}$ & $X\left(X^{2}+Y^{2}\right)$ & $Y\left(X^{2}+Y^{2}\right)$ \\
\hline
\end{tabular}

freedom necessary to describe the whole displacement field has been reduced from typically thousands in local or standard global approaches to eleven in the present method.

Let us denote with $\mathrm{a}^{-}$oversign the amplitudes of uncentered distortion am- 
plitudes, then Table 2 can be rewritten as the linear transform

$$
\left(\begin{array}{c}
\bar{u}_{0} \\
\bar{v}_{0} \\
\bar{u}_{1 x} \\
\bar{v}_{1 x} \\
\bar{u}_{1 y} \\
\bar{v}_{1 y} \\
\bar{d}_{1} \\
\bar{d}_{2} \\
\bar{p}_{1} \\
\bar{p}_{2} \\
\bar{r}_{1}
\end{array}\right)=\boldsymbol{T}\left(\begin{array}{c}
u_{0} \\
v_{0} \\
u_{1 x} \\
v_{1 x} \\
u_{1 y} \\
v_{1 y} \\
d_{1} \\
d_{2} \\
p_{1} \\
p_{2} \\
r_{1}
\end{array}\right)
$$

where $\boldsymbol{T}$ is composed of four blocks

$$
\boldsymbol{T}=\left(\begin{array}{cc}
\boldsymbol{I} & \boldsymbol{T}_{1} \\
\mathbf{0}_{5,6} & \boldsymbol{T}_{2}
\end{array}\right)
$$

with

$$
\boldsymbol{T}_{1}=\left(\begin{array}{ccccc}
\left(3 X_{c}^{2}+Y_{c}^{2}\right) & 2 X_{c} Y_{c} & \left(X_{c}^{2}+Y_{c}^{2}\right) & 0 & -\left(X_{c}^{3}+X_{c} Y_{c}^{2}\right) \\
2 X_{c} Y_{c} & \left(X_{c}^{2}+3 Y_{c}^{2}\right) & 0 & \left(X_{c}^{2}+Y_{c}^{2}\right) & -\left(X_{c}^{2} Y_{c}+Y_{c}^{3}\right) \\
-6 X_{c} & -2 Y_{c} & -2 X_{c} & 0 & \left(3 X_{c}^{2}+Y_{c}^{2}\right) \\
-2 Y_{c} & -2 X_{c} & 0 & -2 X_{c} & 2 X_{c} Y_{c} \\
-2 Y_{c} & -2 X_{c} & -2 Y_{c} & 0 & 2 X_{c} Y_{c} \\
-2 X_{c} & -6 Y_{c} & 0 & -2 Y_{c} & \left(X_{c}^{2}+3 Y_{c}^{2}\right)
\end{array}\right)
$$

and

$$
\boldsymbol{T}_{2}=\left(\begin{array}{ccccc}
1 & 0 & 0 & 0 & -X_{c} \\
0 & 1 & 0 & 0 & -Y_{c} \\
0 & 0 & 1 & 0 & 0 \\
0 & 0 & 0 & 1 & 0 \\
0 & 0 & 0 & 0 & 1
\end{array}\right)
$$


The above correspondence shows in particular that a radial distortion centered at $\boldsymbol{x}_{c}$ does generate two decentering corrections. Conversely, it is appropriate to define the distortion center as the point $\boldsymbol{x}_{c}$ such that the decentering corrections vanish. The procedure then simply consists of evaluating the above amplitudes, $\bar{d}_{1}, \bar{d}_{2}$ and $\bar{r}_{1}$ by digital image correlation, and compute the effective distortion center as

$$
\begin{aligned}
& x_{c} \equiv-\left(\bar{d}_{1} / \bar{r}_{1}\right) L \\
& y_{c} \equiv-\left(\bar{d}_{2} / \bar{r}_{1}\right) L
\end{aligned}
$$

so that one can easily check that $d_{1}=d_{2}=0$. The optical distortion can thus finally be captured by the following intrinsic 7 parameters, namely, mean deviatoric strain (to be computed from $\boldsymbol{u}_{1 x}$ and $\boldsymbol{u}_{1 y}$ ) (i.e., 2 parameters), paraxial corrections (i.e., 2 parameters), radial correction (i.e., 1 parameter) and the position of the distortion center (i.e., 2 parameters). From the above 11 variables introduced, three correspond to arbitrary rigid body motions, and a fourth one sets the magnification factor.

\subsection{Artificial Test Case}

To check that the proposed approach does actually measure distortion fields, a numerical test case has been implemented. It consists of applying a distortion field to the dot centers of the numerically generated target and uses I-DIC between this deformed picture and the reference one. Two different pictures have been generated (Figure 3), one of them with global gray level variations and the other with no variation. The variation is an artificial shadow created by adding a gray level proportional to the distance from the upper left corner of the image to each pixel. In the example used herein, the difference between the gray level of the upper left and the bottom right corner is 30 (i.e., from 0 to 30 gray levels for black pixels, and from 225 to 255 for the white pixels).

The RMS error between the prescribed and the measured distortion field is $3 \times 10^{-2}$ pixel in both cases. The main cause explaining these values is related to 


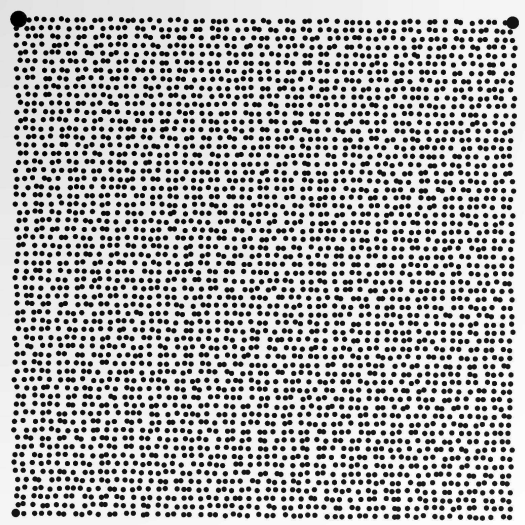

(a)

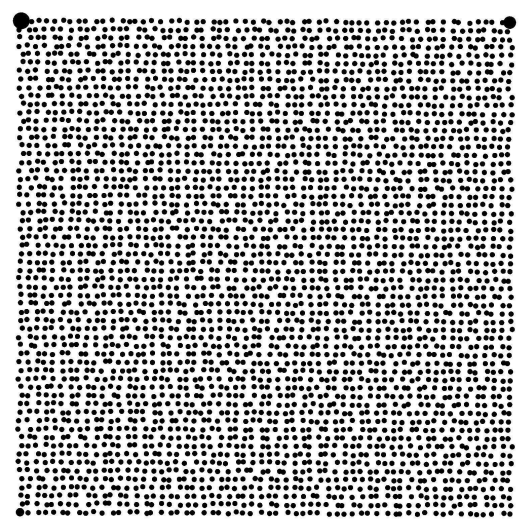

(b)

Figure 3: Numerically deformed calibration pattern used for the artificial test case: (a) with gray level gradient, (b) with no variation

the binarization of the images, and not to the non uniformity of the gray levels. The corresponding error on the distortion coefficients is less than $6 \times 10^{-2}$ pixel. This value is deemed sufficient for distortion analyses. The positioning error of the center is about 1 pixel in the $x$-direction and 3 pixels in the $y$-direction as shown in Figure 4. This small value validates the proposed procedure.

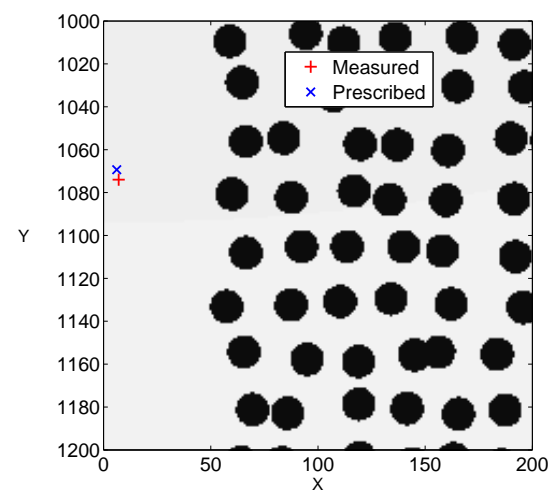

Figure 4: Position of the distortion center. In red the prescribed center and in blue the calculated one. 


\section{Analysis}

In this section, discussions about the proposed method will be developed. Moreover, a comparison with other procedures $[21,12,8,10,11,4,22]$ and an analysis on an a priori resolution estimation [14] will be performed. A Canon EOS 60D camera is used herein with a 50-mm Optimus lens. The definition of the pictures is $1662 \times 1662$ pixels. The picture is acquired in RAW format. Each group of four pixels is then used to calculate the corresponding gray level pixel that ranges from 0 to 255 using standard RGB to grayscale conversion formula [23].

Since the reference configuration is purely numerical, a first procedure is required to make the gray level registration easier. Furthermore, to avoid lighting and reflection issues, a set of pictures has been segmented to obtain binary copies. The present procedure uses Otsu's method [24] to calculate the threshold. It consists of assuming that the image contains two classes of pixels (i.e., white and black) and then calculating the threshold that minimizes the intraclass variance. Moreover, to avoid the sharp edge effects of the black dots, the images are convolved with a Gaussian filter to smoothen the interfaces between black dots and the white background. This Gaussian filter has a 5-pixel diameter.

\subsection{Integrated-DIC}

The correlation operation is performed between these pictures and leads to a displacement field. The reliability of the method is evaluated by the gray level error field (or correlation residual), which is computed at any pixel location as the deviation from the gray level conservation

$$
\epsilon(\boldsymbol{x})=\left|f(\boldsymbol{x})-g\left(\boldsymbol{x}+\boldsymbol{u}_{m}(\boldsymbol{x})\right)\right|
$$

where $\boldsymbol{u}_{m}$ is the measured displacement field. 
Figures 5(a-b) show the distortion fields obtained by I-DIC with an etched target. In this case, the distortion field is easily extracted from the results because of the formulation used. The physical size of one pixel is about $90 \mu \mathrm{m}$ (this value is obtained by measuring the pixel size of the target on the picture and divide it by its actual length, which was evaluated with a caliper) The maximum amplitude of the displacement field is equal to 7 pixels. As expected, the distortion levels are larger at the corners. A residual error map (in gray levels) is shown in Figure 5(c). It proves that the random pattern was properly registered, and that the sharpest parts of the picture (dot boundaries) revealed large error values. These errors on the dot boundaries come from the comparison of a numerical target with rather sharp dot edges and a real picture shot by a camera (dot boundaries are smoother). This effect can be reduced by processing pictures (segmentation and convolution by a Gaussian filter as earlier mentioned).

\subsubsection{Resolution Estimation}

To estimate the resolution of the distortion measurements, several pictures of the same etched calibration target are taken. The correlation procedure between the numerical reference and each of these images results in the position of the center of the radial distortion, a distortion field $\boldsymbol{u}(\boldsymbol{x})$ and a set of distortion coefficients (see Section 2.2).

The mean and the standard deviation of these values are calculated to compare the results. A reference displacement field is estimated as the average of the 10 measured displacement fields. Then, a root mean square (RMS) average is computed on the difference between a displacement field and the reference solution. Figure 6 shows the change of the RMS average in both directions for the 10 analyzed pictures. The RMS difference between consecutive displacement measurements is $2.5 \times 10^{-3}$ pixel for the displacement field. This level is very 


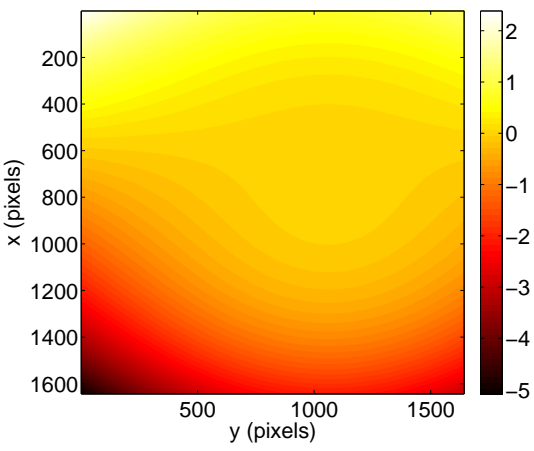

(a)

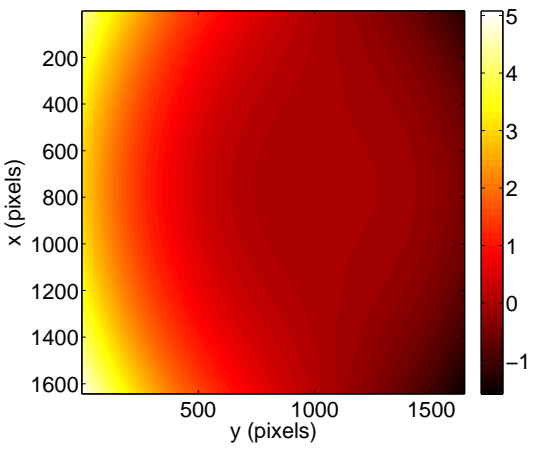

(b)

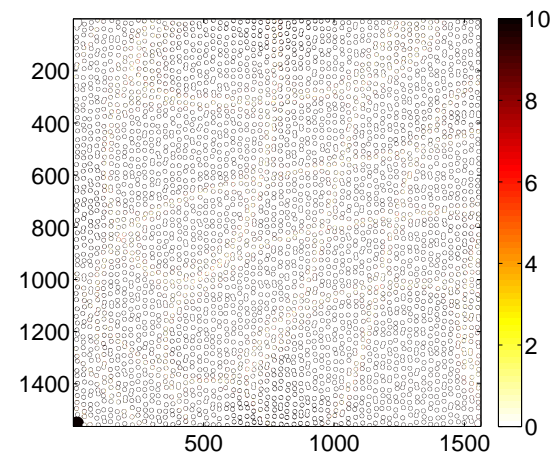

(c)

Figure 5: Example of distortion field (a-b) expressed in pixels, and gray level error (c). The digitization of the picture of the etched target is 8 bits. The missing dot (left corner, see Figure 2) leads to very high residuals

low. It can be achieved only thanks to the integrated approach used herein.

In the proposed setting, the position of the effective center of distortion is part of the unknowns. Figure 7 shows the coordinates found for the 10 pictures. The position of the center belongs to a box whose size is of the order of 1 pixel (RMS difference: 0.65 pixel and 0.43 pixel along each direction). This value is very small and illustrates the robustness of the evaluation of the distortion center.

This a posteriori resolution analysis can be related to the a priori estimates 


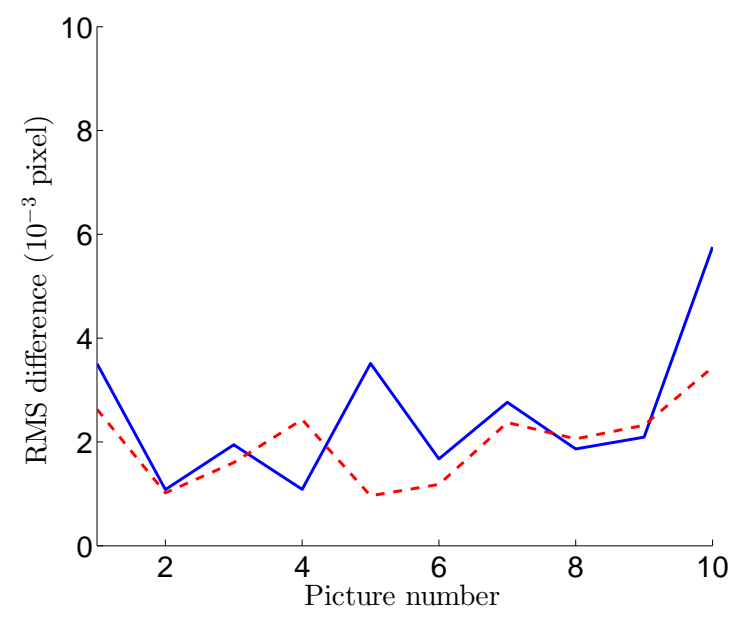

Figure 6: RMS average on displacement (induced by distortions only) difference along the 2 considered directions $\boldsymbol{e}_{x}$ (solid line) and $\boldsymbol{e}_{y}$ (dashed line) for a series of ten pictures of the etched target

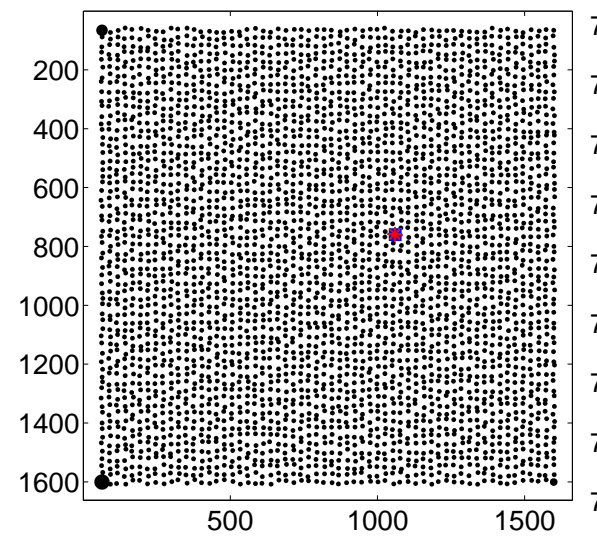

(a)

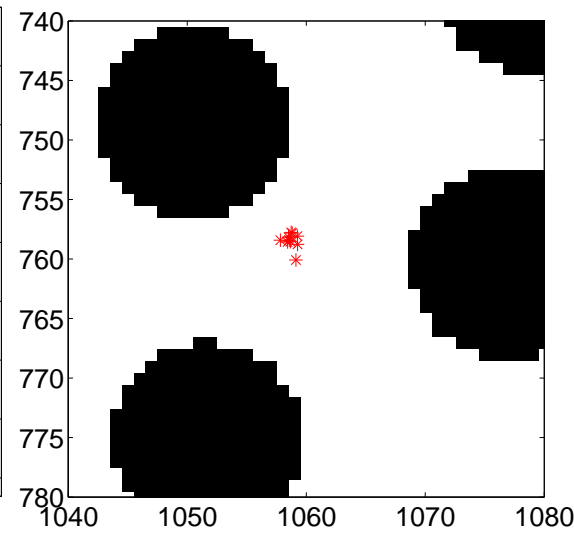

(b)

Figure 7: Position of the distortion center on the reference picture (a) and on the zoom (b)

introduced in Section 3.2. Performing this calculation in the present case leads to an evaluation of the error on each unknown coefficient. The a priori estimation of the uncertainties is shown in Figure 8(a) and the actual covariance matrix is presented in Figure 8(b). The same order of magnitude is observed in both cases. 
The error on $r_{1}$ parameter (i.e., corresponding to the radial distortion) is larger than the others, which can be explained by its influence on the measurement (i.e., the radial field is the most important one [3]).

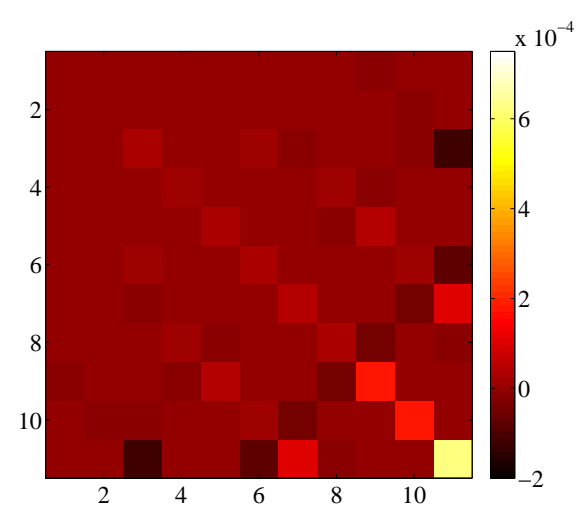

(a)

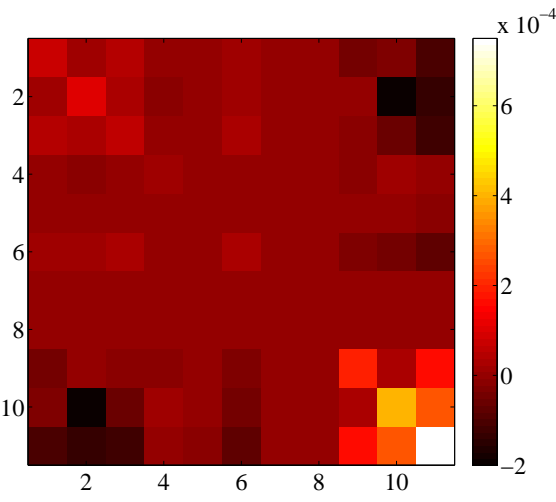

(b)

Figure 8: Covariance matrix (expressed in pixel $^{2}$ ) obtained from the a priori estimation (a) and with the correlation calculation (b). Each component of the covariance matrix is represented by its magnitude

It is important to note that the present estimation of the uncertainties does not take in account the procedure used to search for the center of the radial distortions. This approximation can explain the difference of standard resolution level on the fields except the radial one. By performing the same analysis with a higher degree of representation for the radial distortions, the same magnitude is observed for the new coefficients, but their level of resolution was ten times higher.

\subsubsection{Repeatability of the measurement}

Distortion fields are mainly determined by the sensor-lens pair. A given camera with a given lens setting will yield a specific distortion field. This work focuses on the uncertainties due to the repositioning of the lens on the camera with the etched target. Between each image, the lens has been totally removed 
and repositioned on the camera while the other settings remained unchanged. Two sets of pictures have been shot, the camera has been rotated by a small angle between the two sets. In this calculation, only the first five pictures have been segmented, the others are the raw acquisitions.

As in the previous case, the mean and the standard deviation over these values are calculated to compare the results. A reference calculation is obtained for each set of 5 pictures (one for the first five and a second one for the pictures with the second camera orientation) as the mean of the 5 displacement fields along $\boldsymbol{e}(x)$ (respectively $\boldsymbol{e}(y)$ ). Again, an RMS calculation is performed with the difference between a displacement field and its respective reference. Figure 9 shows the change of RMS values for the 10 pictures. It is impossible to see a clear difference between the two groups of pictures. The segmentation does not appear to have any influence on the accuracy of the registration. The main effect is due to the fact that the lens is fully disassembled from the camera. When compared to the previous case (Figure 6), the standard uncertainties of the measured displacement field has increased by a factor close to 4 on average. The present level, of the order of $10^{-2}$ pixel, still remains very low.

As for the previous case, Figure 10 shows the coordinate locations found for the distortion center for the 10 pictures. The position of the distortion center varies more (close to a factor 5) and the positioning error has become as large as the displacement values. Two different groups of position are obtained corresponding to the two sets of pictures. The segmentation does not appear to have any influence on the accuracy of the positioning.

To quantify the difference induced by the binarization, a calculation on the same image with and without segmentation has been performed. Figure 11 shows the differences between the displacement field obtained with and without segmentation. The main differences are located on the edges of the picture. When considering the RMS average, the following values are obtained: 0.016 


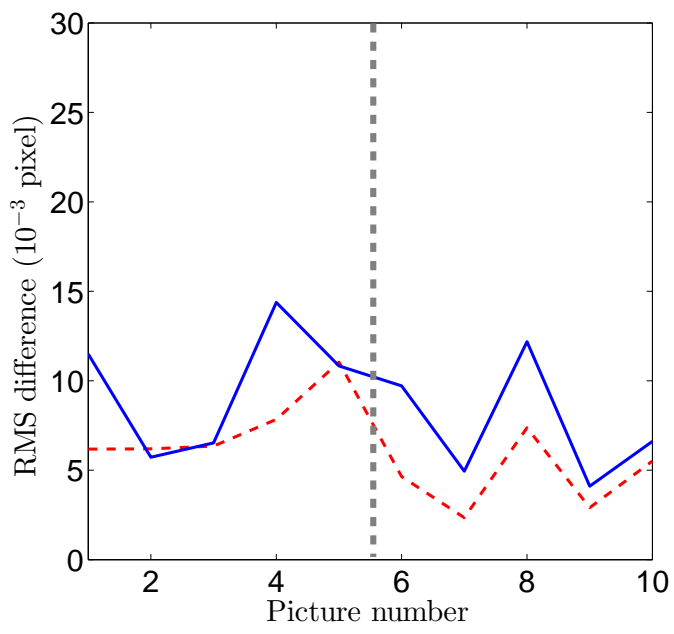

Figure 9: RMS average on displacement (induced by distortions only) difference along the 2 considered directions $\boldsymbol{e}_{x}$ (solid line) and $\boldsymbol{e}_{y}$ (red dashed line) for a series of $2 \times 5$ lens removals

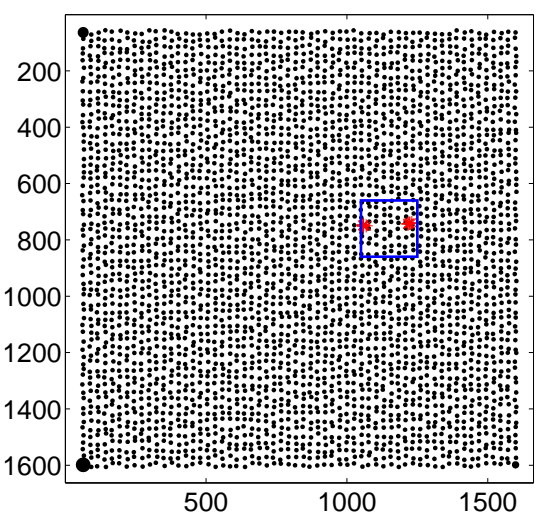

(a)

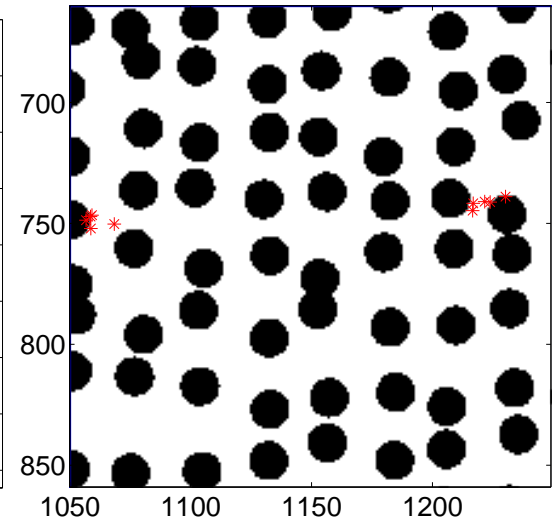

(b)

Figure 10: Position of the distortion center on the reference picture with lens removal (a). The blue box corresponds to the zoom (b). The two sets of pictures are clearly visible

and 0.012 pixel along both directions. This level is slightly higher than what was observed in the previous analyses (Figure 9). However, it is worth remembering that reflection artifacts are present in that case.

Figure 12 shows the impact on the position of the center of the distortion. 


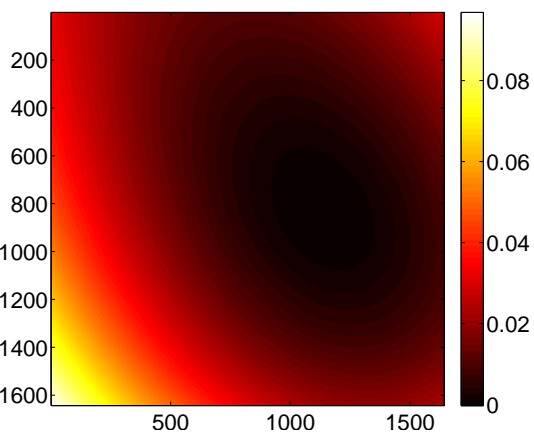

(a)

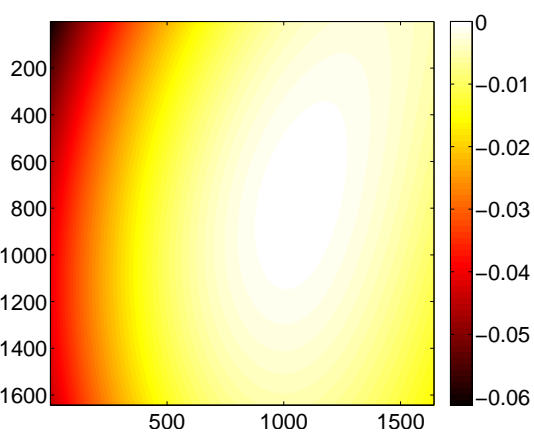

(b)

Figure 11: Difference between the displacement field (expressed in pixels) obtained from a segmented and a raw picture along $\boldsymbol{e}_{x}$ (a) and $\boldsymbol{e}_{y}$ (b) directions

A positioning error appears between the two images, which has the same order of magnitude as the variations observed previously (Figure 10).

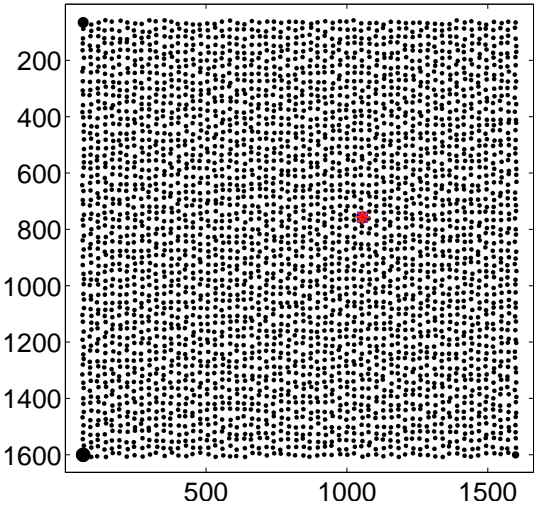

(a)

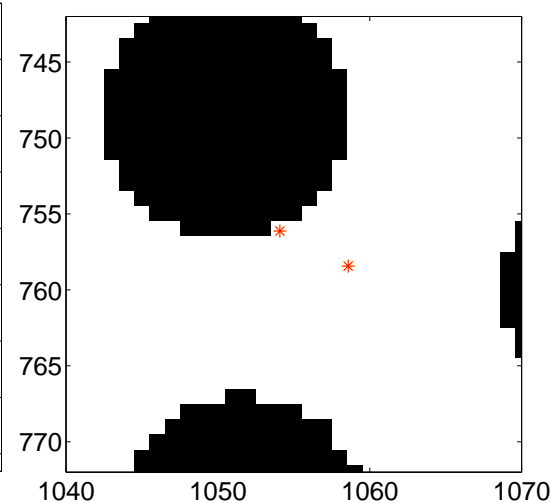

(b)

Figure 12: Position of the calculated center of the radial distortion for a segmented and a raw picture (a). Zoom on the interesting area (b)

\subsection{Spline Basis Functions}

Several methods exist to evaluate and correct for optical distortions (see Section 1). In the sequel, a spline-based kinematics to describe the distortions [12] 
will be used on the etched target and compared to the previous results. The comparison is performed with a third-order and fifth-order spline representation. It follows that $16 \times 2$ and $36 \times 2$ degrees of freedom are considered, which are at least three times as many as in the integrated approach. The mean residual error with the present approach (i.e., $5.6 \%$ and $5.1 \%$ of the gray-level dynamic range) is lower when compared with the integrated approach, which is to be expected since the number of degrees of freedom has increased, but remains close to the previous value (i.e., 6.3\%). These (rather) high values are due to the fact that a synthetic reference image is considered herein.

Figures 13(a-b) show the distortion fields obtained by the spline basis with the printed target. In that case, the method does not allow for the separation between rigid body motions and the distortion field. The residual error map shown in Figure 13(c) proves that the random pattern was properly registered.

Two different displacement fields are measured with the spline decomposition. Figures 14 and 15 show the difference between those displacement fields along the two directions with those obtained with the parametric description. Large differences are observed on the boundary, but the central values are similar with all methods. The RMS differences between the displacement fields found with the parametric model and the third-order spline basis method are equal to 0.68 pixel along $\boldsymbol{e}_{x}$ and 0.39 pixel along $\boldsymbol{e}_{y}$ (0.66 pixel along $\boldsymbol{e}_{x}$ and 0.44 pixel along $\boldsymbol{e}_{y}$ for the fifth order spline basis). These differences can be attributed to the manufacturing process of the target, since when performing the same operation with a printed target (Figure 16) instead of an etched one, these differences are reduced by a factor close to 10 . This observation stresses the fact that only global distortions are measured. The decomposition into optical and printing (or etching) contribution has not been addressed herein. Thus, if this procedure is to be used to measure the optical distortion, the calibration target 


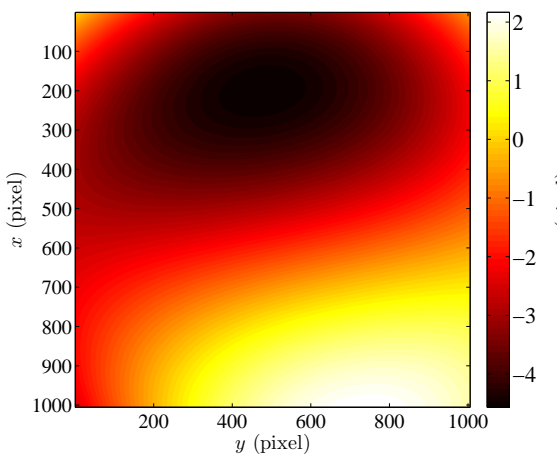

(a)

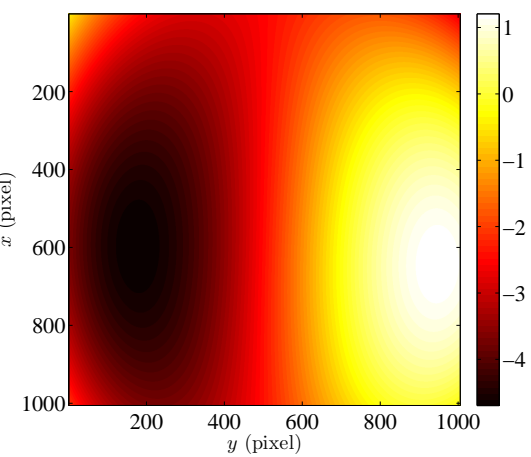

(b)

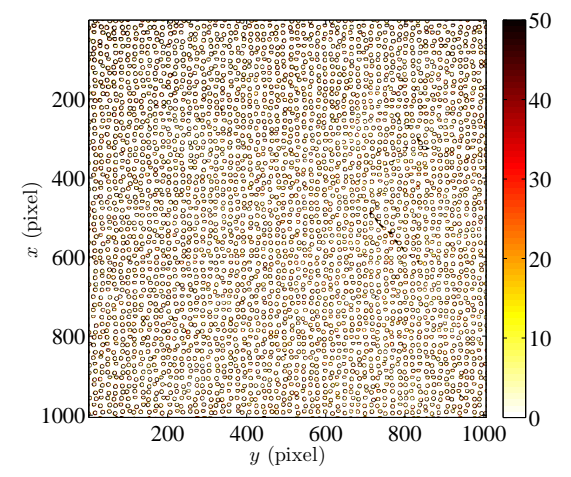

(c)

Figure 13: Example of displacement field ( $\mathrm{a}-\mathrm{b})$ expressed in pixels, and gray level error (c) for the printed calibration target

should be printed with high fidelity.

Differences between the spline approach and the parametric description increase with the spline degree as could be anticipated. The parametric description seems to be effective since with half as much degrees of freedom as the spline approach, it is possible to closely describe the whole distortion field. Moreover, the use of two different descriptions allows us to compare the fields and use this comparison to cross-validate the truncation of the two bases.

The main drawback of this non-parametric approach is that rigid body motions, magnification changes and distortion fields are coupled and cannot be 


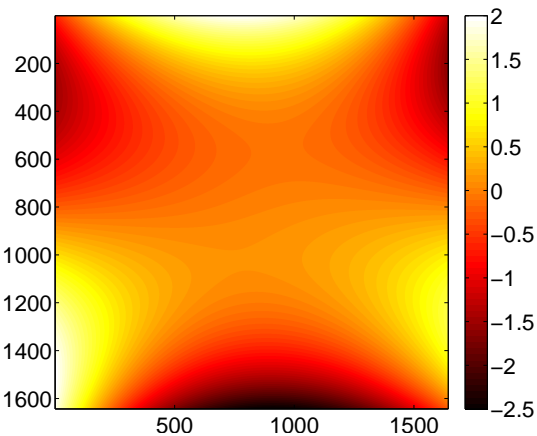

(a)

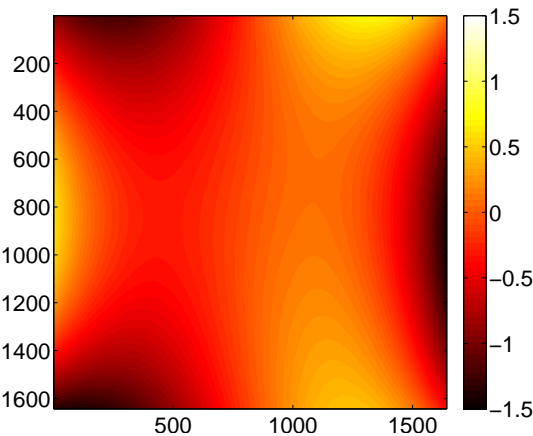

(b)

Figure 14: Difference between the displacement field (expressed in pixels) for a third-order spline basis and the parametric model along $\boldsymbol{e}_{x}$ (a) and $\boldsymbol{e}_{y}$ (b) corresponding to an RMS difference of 0.68 pixel and 0.39 pixel in both directions with an etched target

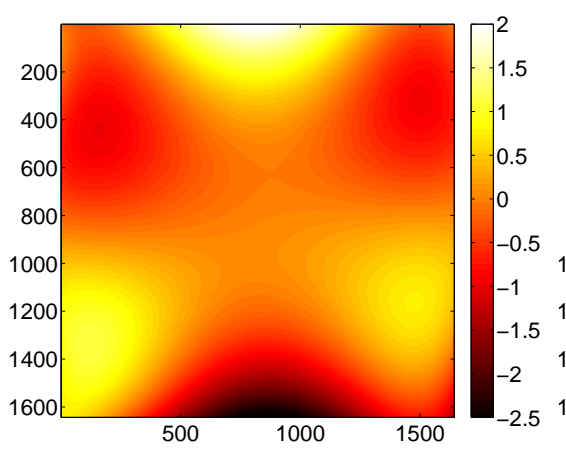

(a)

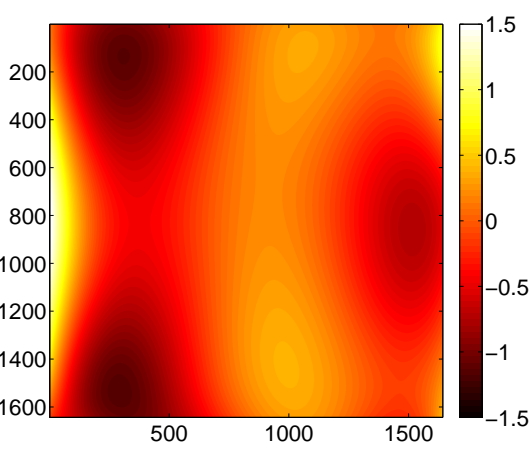

(b)

Figure 15: Difference between the displacement field (expressed in pixels) for a fifth-order spline basis and the parametric model along $\boldsymbol{e}_{x}$ (a) and $\boldsymbol{e}_{y}$ (b) corresponding to an RMS difference of 0.66 pixel and 0.44 pixel in both directions with an etched target

separated without an additional post-processing step. Moreover, it means that the position of the calibration target has to be perfect with respect to the camera (e.g., perpendicularity, centering) to represent only the distortion field. Otherwise the apparent center is not properly estimated.

A resolution analysis is also performed with the spline-based calculation by using the method presented above. Figure 17 shows the covariance matrix 


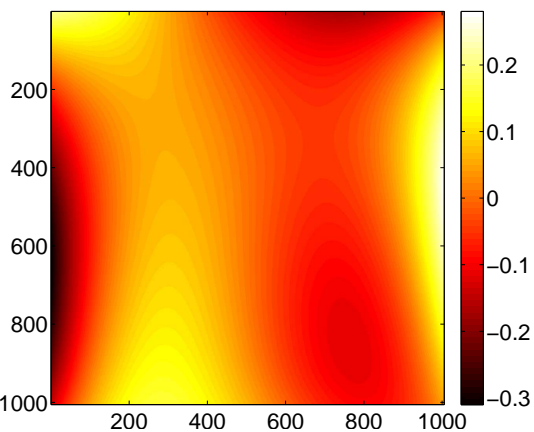

(a)

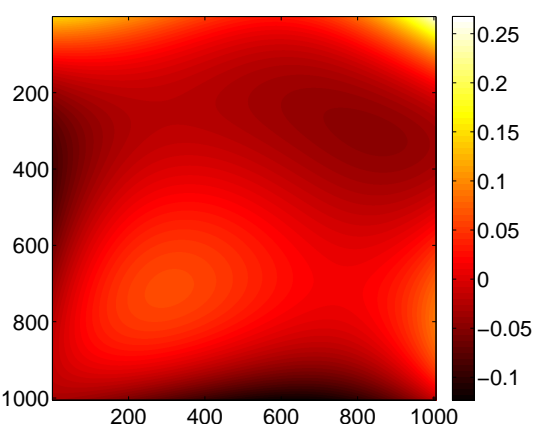

(b)

Figure 16: Difference between the displacement field (expressed in pixels) for a third-order spline basis and the parametric model along $\boldsymbol{e}_{x}$ (a) and $\boldsymbol{e}_{y}$ (b) corresponding to an RMS difference of 0.08 pixel and 0.04 pixel in both directions with a printed target

for third order basis along $\boldsymbol{e}_{x}$. The most uncertain parameters are the values

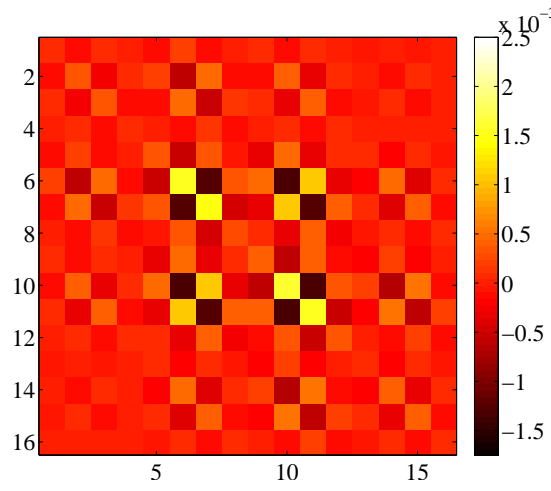

(a)

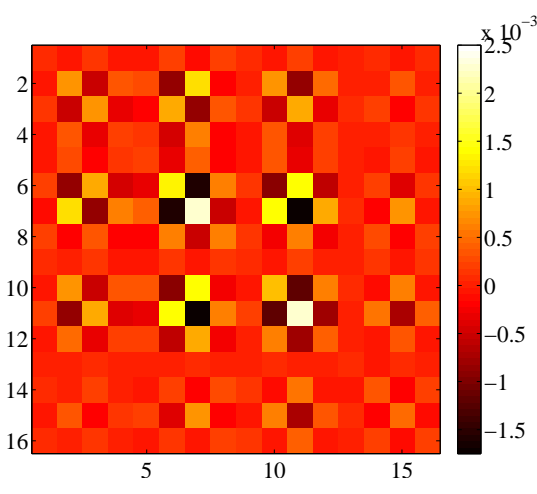

(b)

Figure 17: Covariance obtained from the a priori estimation (a) and the correlation calculation (b). Each component of the covariance matrix is represented by its magnitude

corresponding to the center area of the field in both case. A very good agreement is observed for the a priori estimate of the covariance matrix, when compared to its experimental evaluation. 


\section{Conclusions}

This study introduces a new method to evaluate global distortions (i.e., coming from optical imaging and printing of known calibration target). First, an artificial random texture has been printed on an aluminium sheet. Second, a steel plate has been etched with randomly generated pattern of dots from a numerical reference. Therefore, in both cases the reference is a synthetic picture.

The calibration target is imaged and the corresponding picture is registered with the numerical reference. The registration is based upon digital image correlation. The first procedure corresponds to an integrated approach in which the distortion fields, which are defined with a few degrees of freedom, are directly measured. Contrary to most techniques used for the correction of distortions, which are geometrical methods, the present approach is solely based on digital image correlation.

The proposed methodology provides a natural definition of the effective distortion center position such that the decentering distortion field is exactly canceled. This definition enables for an intrinsic characterization of the distortion field.

As all measurement techniques, the present method implies uncertainties. Some are due to the optical model (i.e., first order approximation, Taylor truncation), to the correlation procedure (e.g., gray level interpolation) or the imaging device itself (e.g., acquisition noise, vibration during picture acquisition). The error due to acquisition noise is evaluated with two different methods. The first one consists of taking many pictures of the same scene and measuring the scatter of the results. These results can be compared with an a priori resolution analysis, which provides the performance of the DIC technique based on a propagation of uncertainties. The uncertainties found in both cases are very close to each other and their level is very low as they benefit from the integrated 
DIC approach.

Once the distortion field has been estimated, it can be accounted for in the picture to create a 'distortion-free' image. Using an integrated approach to DIC enables only a few parameters to be determined to describe the entire field and to improve the uncertainties on these coefficients. Using a parametric approach gives the opportunity to decompose the results in terms of referential dependent fields and intrinsic optical distortions.

This technique has many possible applications. It can be used to precalibrate cameras in a stereoDIC method to separate the distortion corrections and the stereo-system calibration. More generally, it can be used to suppress (or limit) the influence of the distortion in many DIC measurements (e.g., in optical or electron microscopy [19]). As taking a picture of the calibration target and calculating the distortion parameters are fast steps, this technique remains simple and easy to implement prior to or after any mechanical test. However, special care should be exercised to check the quality of physical reproduction by printing or etching of the calibration target. 


\section{References}

[1] D. C. Brown. Decentering distortion of lenses. Photogrammetric Engineering, 32(3):444-462, 1966.

[2] D. C. Brown. Close-range camera calibration. Photogrammetric Engineering, 37(8):855-866, 1971.

[3] M. A. Sutton, S. R. McNeill, J. D. Helm, and Y. J. Chao. Photomechanics, Topics in Applied Physics, chapter Advances in two-dimensional and threedimensional computer vision. Springer, New York, USA, 2000.

[4] S Yoneyama, H Kikuta, A Kitagawa, and K Kitamura. Lens distortion correction for digital image correlation by measuring rigid body displacement. Optical Engineering, 45(2), 2006.

[5] M. A. Sutton, J.-J. Orteu, and H W. Schreier. Image correlation for shape, motion and deformation measurements. Springer, 2009.

[6] P. Lava, W. Van Paepegem, S. Coppieters, I. De Baere, Y. Wang, and D. Debruyne. Impact of lens distortions on strain measurements obtained with 2d digital image correlation. Opt. Lasers Eng., 51:576-584, 2013.

[7] B. Pan, L. Yu, D. Wu, and L. Tang. Systematic errors in two-dimensional digital image correlation due to lens distortion. Optics Lasers Eng., 51(2):140-147, 2013.

[8] J. Weng, P. Cohen, and M. Herniou. Camera calibration with distortion models and accuracy evaluation. IEEE Transactions on pattern analysis and machine intelligence, 40(10):965-980, 1992.

[9] O. Faugeras. Three-dimensional computer vision: a geometric viewpoint. MIT Press, Cambridge, MA, USA, 1993. 
[10] Z. Zhang. A flexible new technique for camera calibration. IEEE Transactions on Pattern Analysis and Machine Intelligence, 22(11):1330-1334, 2000.

[11] C. V. Vincent and T. Tjahjadi. Multiview camera-calibration framework for nonparametric distortions removal. IEEE Transactions on Robotics, 21(5):1004-1009, 2005.

[12] A. Goshtasby. Correction of image deformation from lens distortion using bezier patches. Computer Vision, Graphics, and Image Processing, 1989.

[13] C. Harris and S. Stephens. A combined corner and edge detector. In Proceedings of the 4th Alvey Vision conference, pages 147-151, 1988.

[14] F. Hild and S. Roux. Digital image correlation. In P. Rastogi and E. Hack, editors, Optical methods for solid mechanics, chapter 5, pages 183-228. Wiley-VCH, 2012.

[15] F. Hild, S. Roux, R. Gras, N. Guerrero, M. E. Marante, and J. FlórezLópez. Displacement measurement technique for beam kinematics. Optics and Lasers in Engineering, 47:495-503, 2009.

[16] J. Réthoré, T. Elguedj, P. Simon, and M. Coret. On the use of nurbs functions for displacement derivatives measurement by digital image correlation. Experimental Mechanics, 50:1099-1116, 2009.

[17] F. Hild and S. Roux. Digital image correlation: From measurement to identification of elastic properties - a review. Strain, 42:69-80, 2006.

[18] S. Roux and F. Hild. Stress intensity factor measurements from digital image correlation: post-processing and integrated approaches. International Journal of Fracture, 140:141-157, 2006. 
[19] A. Guery, F. Latourte, F. Hild, and S. Roux. Characterization of sem speckle pattern marking and imaging distortion by digital image correlation. Meas. Sci. Technol., 25(1):015401, 2014.

[20] International Organization for Standardization, Geneva (Switzerland). International Vocabulary of Metrology - Basic and general Concepts and Associated Terms, 2007.

[21] W. B. Green, P. L. Jepsen, J. E. Kreznar, R. M. Ruiz, A. A. Schwartz, , and J. B. Seidman. Removal of instrument signature from mariner 9 television images of mars. Applied Optics, 14:105-114, 1975.

[22] P. Lava, W. Van Paepegem, S. Coppieters, I. de Baere, and D. Debruyne. Impact of lens distortions on strain measurements obtained by digital image correlation. In SEM XII International Congress and Exposition on Experimental and Applied Mechanics, 2012.

[23] International Telecommunication Union. Studio Encoding Parameters of Digital Television for Standard 4:3 and Wide-screen 16:9 Aspect Ratios: Recommendation ITU-R BT.601-6: (Question ITU-R 1/6). International Telecommunication Union, 2007.

[24] N. Otsu. Threshold selection method from gray-level histograms. IEEE Transactions on Systems Man and Cybernetics, 9(1):62-66, 1979. 


\section{Appendix: Effect of Rigid Motions of the Target on the Kinematic}

\section{Basis}

The following analysis aims at discussing the effect of slight mispositionings of the calibration target on the apparent motions in the image plane of the camera sensor. The latter is assumed to be unskewed. The reference configuration corresponds to the situation in which the optical axis of the camera is perfectly aligned with the normal to the target plane. Without loss of generality, these two axes are aligned with the $\boldsymbol{z}$ direction. The so-called pinhole camera model is used hereafter. Under these hypotheses, the pixel coordinates $\mathbf{p}\left(x_{r}, y_{r}\right)$ of any point $\mathbf{P}\left(X_{r}, Y_{r}, 0\right)$ belonging to the calibration target reads (Figure 18)

$$
\left\{\begin{array}{c}
x_{r} \\
y_{r}
\end{array}\right\}=\left\{\begin{array}{c}
\frac{f_{x}}{d_{z}} X_{r} \\
\frac{f_{y}}{d_{z}} Y_{r}
\end{array}\right\}
$$

where $d_{z}$ denotes the distance between the optical center of the camera and the center of the calibration target, and $f_{x}, f_{y}$ parameters including the scale factors and the focal length of the pinhole system [5]. In the present case, $d_{z}=500 \mathrm{~mm}$, $f_{x}=f_{y}=5 \times 10^{3}$ pixels. This first transformation allows the physical size of one pixel to be determined.

Let us assume that there are misalignments with respect to the reference configuration. Let $d_{x}$ and $d_{y}$ denote the in-plane displacements, and $R_{i j}$ the components of the rotation matrix describing the three rigid body rotations. These rigid body motions induce apparent motions in the image plane so that the new (pixel) coordinates read

$$
\left\{\begin{array}{c}
x_{n} \\
y_{n}
\end{array}\right\}=\left\{\begin{array}{c}
c_{x}+f_{x} \frac{R_{11} X_{r}+R_{12} Y_{r}+d_{x}}{R_{3} X_{r}+R_{32} Y_{r}+d_{z}} \\
c_{y}+f_{y} \frac{R_{21} X_{r}+R_{22} Y_{r}+d_{y}}{R_{31} X_{r}+R_{32} Y_{r}+d_{z}}
\end{array}\right\}
$$

where $c_{x}, c_{y}$ corresponds to the decentering of the optical axis. The magnitude of the angular offsets are assumed to be small so that only first order terms are considered (i.e., $\left.\left.\left(R_{31} X_{r}+R_{32} Y_{r}\right) \ll d_{z}\right), \cos \theta \approx 1, \sin \theta \approx \theta\right)$. This assumption 


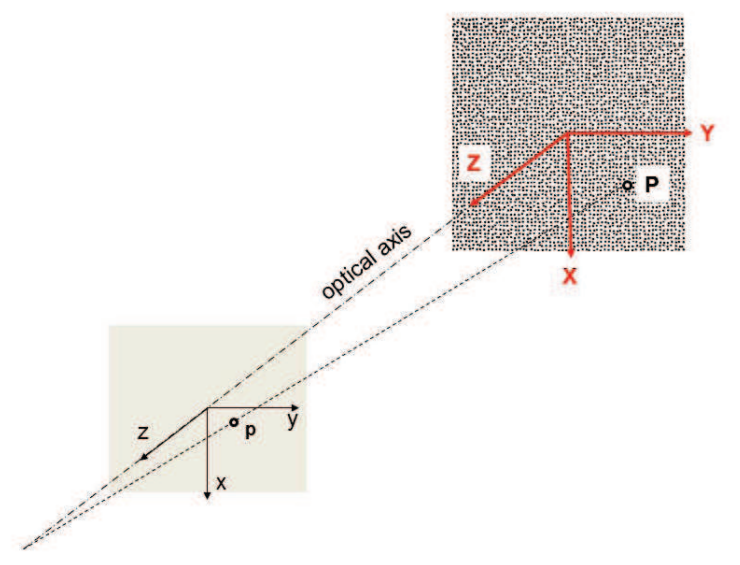

Figure 18: Front image plane construction of the pixel location $\mathbf{p}\left(x_{r}, y_{r}\right)$ of any point $\mathbf{P}\left(X_{r}, Y_{r}, 0\right)$ of the calibration target

is generally not made when performing bundle adjustments [5]. However, it is assumed to be a very good approximation in the present case. In the sequel, the rotation matrix is based upon the choice of Cardan angles $\left(\theta_{1}, \theta_{2}, \theta_{3}\right)$ to describe the frame changes induced by the previous rigid body motions. By using the so-called $x-y-z$ combination, the rotation matrix reduces to

$$
[\mathbf{R}] \approx\left[\begin{array}{ccc}
1 & -\theta_{1} & \theta_{2} \\
\theta_{1} & 1 & -\theta_{3} \\
-\theta_{2} & \theta_{3} & 1
\end{array}\right]
$$

The apparent pixel displacements are expressed as

$$
\begin{gathered}
\left\{\begin{array}{c}
u_{a} \\
v_{a}
\end{array}\right\}=\left\{\begin{array}{c}
x_{n} \\
y_{n}
\end{array}\right\}-\left\{\begin{array}{c}
x_{r} \\
y_{r}
\end{array}\right\}= \\
\left\{\begin{array}{c}
d_{x}-y_{r} \theta_{1}+\frac{t_{x}}{t_{z}} \theta_{2} x_{r}-\frac{t_{x}}{t_{z}} \theta_{3} y_{r}-\frac{x_{r}}{f_{x}}\left(y_{r} \theta_{3}-x_{r} \theta_{2}\right) \\
d_{y}+x_{r} \theta_{1}+\frac{t_{y}}{t_{z}} \theta_{2} x_{r}-\frac{t_{y}}{t_{z}} \theta_{3} y_{r}-\frac{y_{r}}{f_{y}}\left(y_{r} \theta_{3}-x_{r} \theta_{2}\right)
\end{array}\right\}
\end{gathered}
$$


Since $\left|x_{0}\right|,\left|y_{0}\right|<750$ pixels, it follows that $\left|x_{0} / f_{x}\right|,\left|y_{0} / f_{y}\right|<0.15$. The parabolic terms $x_{0} y_{0} \theta_{2} / f_{x}$ and $y_{0}^{2} \theta_{2} / f_{y}$ are therefore one order of magnitude less than the linear terms $y_{0}\left(\theta_{1}+\theta_{3}\right)$ and $x_{0}\left(\theta_{1}+\theta_{3}\right)$. Therefore, linear displacement interpolations account for magnification changes and small misalignments of the camera with respect to the calibration target. 


\section{List of Figures}

1 Numerical reference (a) and picture of the printed calibration target (b). It can be noted that there are some defaults due to the printer . . . . . . . . . . . . . . . . . 8

2 Numerical reference (a) and picture of the etched calibration target (b). It can be noted that the large dot on the lower left corner was not etched . . . . . . . . . . . . . . . . . . .

3 Numerically deformed calibration pattern used for the artificial test case: (a) with gray level gradient, (b) with no variation . . . 16

4 Position of the distortion center. In red the prescribed center and in blue the calculated one. . . . . . . . . . . . . . . . 16

5 Example of distortion field (a-b) expressed in pixels, and gray level error (c). The digitization of the picture of the etched target is 8 bits. The missing dot (left corner, see Figure 2) leads to very high residuals . . . . . . . . . . . . . . . . . . . 19

6 RMS average on displacement (induced by distortions only) difference along the 2 considered directions $\boldsymbol{e}_{x}$ (solid line) and $\boldsymbol{e}_{y}$ (dashed line) for a series of ten pictures of the etched target . . . 20

7 Position of the distortion center on the reference picture (a) and on the zoom $(\mathrm{b}) \ldots \ldots \ldots \ldots$

8 Covariance matrix (expressed in pixel $^{2}$ ) obtained from the a priori estimation (a) and with the correlation calculation (b). Each component of the covariance matrix is represented by its magnitude 21

$9 \quad$ RMS average on displacement (induced by distortions only) difference along the 2 considered directions $\boldsymbol{e}_{x}$ (solid line) and $\boldsymbol{e}_{y}$ (red dashed line) for a series of $2 \times 5$ lens removals . . . . . . 23 
10 Position of the distortion center on the reference picture with lens removal (a). The blue box corresponds to the zoom (b). The two sets of pictures are clearly visible . . . . . . . . . . . . . 23

11 Difference between the displacement field (expressed in pixels) obtained from a segmented and a raw picture along $\boldsymbol{e}_{x}$ (a) and

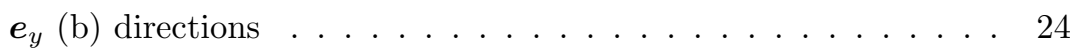

12 Position of the calculated center of the radial distortion for a segmented and a raw picture (a). Zoom on the interesting area (b) 24

13 Example of displacement field (a-b) expressed in pixels, and gray level error $(\mathrm{c})$ for the printed calibration target . . . . . . . . 26

14 Difference between the displacement field (expressed in pixels) for a third-order spline basis and the parametric model along $\boldsymbol{e}_{x}$ (a) and $\boldsymbol{e}_{y}$ (b) corresponding to an RMS difference of 0.68 pixel and 0.39 pixel in both directions with an etched target . . . . . 27

15 Difference between the displacement field (expressed in pixels) for a fifth-order spline basis and the parametric model along $\boldsymbol{e}_{x}$ (a) and $\boldsymbol{e}_{y}$ (b) corresponding to an RMS difference of 0.66 pixel and 0.44 pixel in both directions with an etched target . . . . . 27

16 Difference between the displacement field (expressed in pixels) for a third-order spline basis and the parametric model along $\boldsymbol{e}_{x}$ (a) and $\boldsymbol{e}_{y}$ (b) corresponding to an RMS difference of 0.08 pixel and 0.04 pixel in both directions with a printed target . . . . 28

17 Covariance obtained from the a priori estimation (a) and the correlation calculation (b). Each component of the covariance matrix is represented by its magnitude . . . . . . . . . . 28

18 Front image plane construction of the pixel location $\mathbf{p}\left(x_{r}, y_{r}\right)$ of any point $\mathbf{P}\left(X_{r}, Y_{r}, 0\right)$ of the calibration target $\ldots \ldots \ldots$ 